\title{
Low-cost environmental monitoring mini rover based on IoT technology
}

\author{
Nurul Ayni Mat Pauzi, Seri Mastura Mustaza* and Iskandar Yahya \\ Faculty of Engineering \& Built Environment, Universiti Kebangsaan Malaysia
}

Received: 16-October-2020; Revised: 24-January-2021; Accepted: 25-January-2021

(C)2021 Nurul Ayni Mat Pauzi et al. This is an open access article distributed under the Creative Commons Attribution (CC BY) License, which permits unrestricted use, distribution, and reproduction in any medium, provided the original work is properly cited.

\begin{abstract}
The aim of this research is to construct a rover for environmental condition monitoring, as an alternative for conventional monitoring system and data gathering via the use of closed-circuit $\mathrm{TV}$ (CCTV), personnel and detection animal. The system can be deployed to assess damage and toxicity of areas based on reading from sensors, and for monitoring through visual image from the camera. The rover employs the Internet- of-Things (IoT) concept and is able to monitor the condition of the environment via the help of sensors such as humidity sensor, temperature sensor, air quality sensor, ultrasonic sensor and also with the help of camera. The developed rover will be controlled by a mobile application as the GUI, and the data from the sensor and the camera image can be monitored through the mobile application. If obstacle is detected by the ultrasonic sensor, the mobile application will give notification towards the user through the mobile application, to warn of possible collision. The data from the sensor is sent to the MySQL database and the system is connected via Wi-Fi. The data can be accessed by the user later and can also be used to perform further analysis if needed. This rover is compact in size, able to enter areas that are hard to access, and its usage will lower the cost of monitoring with lower development cost of the rover.
\end{abstract}

\section{Keywords}

Internet of things (IoT), Rover, Mobile application, MySQL.

\section{Introduction}

In this era, monitoring system has become very important not just for security purposes but can serve as an input for smart system development. An effective monitoring system not only capable in continuous monitoring of the surrounding but can also give an early warning in case of emergency or occurrence of undesired event, as well as providing accurate environmental condition at area of interest at any time. Conventional monitoring system such as the use of CCTV and personnel with detection dog or hand-carried sensor devices, have become increasingly outdated and is unsuitable for monitoring large areas, confined or tight spaces such as ventilation ducts [1] and in dangerous or harsh environment such as facilities with high radiation exposure (e.g.: nuclear plant), toxic gas leak or sewers where bacteria and diseases are rampant. This method of monitoring may pose risks of danger, pollution, diseases and other negative effects towards the human and animals involved.

*Author for correspondence

64
Furthermore, the hand-carried devices used can't be deployed over extended period of time for continuous monitoring. The use of CCTVs also has its limitations, where it is inflexible as it cannot monitor large areas, cannot be installed in all areas such as air ventilation system, and when installed in a room, it cannot be moved unless dismantled. Furthermore, CCTV is known to have blind spots. Large number of CCTVs are needed to cover a wide and large area, which increases the investment cost. The cost for installation, purchase and CCTV maintenance also contributes in the high investment cost of this system.

\section{Literature review}

To solve the problems related to conventional CCTV monitoring, researchers have come up with many solutions, and one of it is the use rover or mobile robot [2]. Mobile robots or rovers that are currently available on the market are either too expensive [2] and bulky or inadequate for complete monitoring. Some also rely on laptop or PC to monitor [3]. For example, the work in [4] is designed coastal monitoring [5] designed a mobile robot for mowing, both of which due to its size and application, are well above USD 500. While both of these systems are 
properly equipped with the appropriate sensors, these rovers can't be used to monitor tight and confined spaces such as the air vent and the design is too big. The system in [6-8] is a small and compact rover system but with limited capability whereby there is only one on board sensor and both are not equipped with camera to assist the monitoring activity. Whereas, the system described in $[9,10]$ only have camera but lacks other sensor to monitor the actual condition of the environment. Other compact sized rover such as $[11,7]$ and require the use of laptop or $\mathrm{PC}$ to visualize or operate the rover. To solve the existing problems, the mobile robot or rover needs to be able to monitor the condition of the environment and compact in size to move in locations that is difficult to access and preferably with lower cost. Therefore, instead of posing unnecessary risk towards the life of humans and animals in dangerous environments, this rover can be used as an alternative to pinpoint and assess damaged areas as well as to perform monitoring as preventive maintenance. Furthermore, the gathered data needs to be stored for the purpose of reporting, trend monitoring and further analysis.

This study involves the development of an IoT based, compact sized rover for monitoring of tight, confined spaces, produced at a lower cost, and equipped with sensors for data collection, navigation and camera for surveillance. The IoT technology basically is a system of physical objects that have physical connectivity and communication through IP address between webs to empower gadgets and objects [12]. Therefore, the idea is to have all the onboard sensors, as well as other components of the rover to be connected and available on the internet for fast and real-time remote environmental monitoring. To realize the IoT technology, a mobile application will also be developed as a graphical user interface (GUI) that is able to control the rover and display sensor's data as well as camera image.

\section{Methodology}

The development of the rover is accomplished in four phases. The first phase in the concept design. To identify the specifications of the environmental monitoring mini rover, literature studies have been conducted to identify the weaknesses of conventional surveillance systems and current state-of the-art of environmental monitoring. Focus will be given to the development of a compact sized mobile based rover for accessing spaces that are difficult to access (e.g.: ventilation, air duct, etc.) at a low cost, capable to perform and gather data for a basic monitoring of the environmental surrounding. Cost constraint was also set as the main aim is to produce a low-cost rover. The second phase is the hardware selection and development based on the specification set in the first phase and the third phase is the software development. The software development involved programming of the individual hardware as well as development of the mobile application for manoeuvring the rover and environmental data monitoring. Final phase of the study is the testing phase, whereby the rover is deployed to gather information of its surrounding.

\subsection{Hardware}

As mentioned above, the focus chosen for this mini environmental monitoring rover is for the use in facilities at risk of exposure to radiation, during toxic gas leak, or use in sewage and air vent areas where they can be used for monitoring purposes or to detect damage. The block diagram of the overall IoT based rover system is as shown in Figure 1.

A small size robot is required, as well as a functioning system for detecting air quality and gas concentration, and to detect abnormal temperature changes, as well as cameras to detect damage using image data. Therefore, the rover will be equipped with temperature sensors, air quality sensors and humidity sensor based on the focus of application, as well as ultrasonic sensor to detect obstacles around the robot. Figure 2. shows the individual hardware component of the proposed system. Table 1 list the cost for each of the component. Based on the cost, the rover can be considered low cost with total cost of RM 462.40 (around USD 100).

A Raspberry Pi system is used as the central platform and data transmission to connects the sensors, motors as well as the camera. Raspberry Pi is selected also due to its ability in providing distinctive $\mathrm{Wi}-\mathrm{Fi}$ module. The advantage of this is, as it is a microcomputer with Wi-Fi functionality, it can be used as a server during the system run. Model used in this project is Raspberry $\mathrm{Pi} 3 \mathrm{~B}+$, that is among of the latest model in the market. Arduino also is used in this project as a slave to the Raspberry Pi system, which act as the master. I2C communication is implemented between the Raspberry Pi and Arduino. All the analog sensors (temperature\& humidity and air quality sensors) is connected to the Arduino since the Raspberry Pi does not have the analog input port. All four of the ultrasonic sensors are also connected to the Arduino. The motor driver and Pi camera [10] 
[13] are connected directly on to the Raspberry Pi board.

For the temperature $\&$ humidity sensor, the type used is DHT-11 [14]. It is connected to the GPIO port of Raspberry Pi and Adafruit DHT is used to read both the humidity and temperature values, before displaying the values for monitoring. The DHT-11 would detect any changes in temperature and humidity with resolution of $0.1{ }^{\circ} \mathrm{C}$ and $0.1 \%$, respectively. Another temperature sensor, DS18B20, with a wider detection range compared to the DHT11 was also used, to detect a higher temperature change.

The air quality sensor used in this study is the MQ$135[15,16]$. The MQ-135 sensor is used in this study to detect the presence of venomous gases, and this model specifically is suitable for detecting or measuring of Ammonia (NH3), Mono-Nitrogen Oxide $\left(\mathrm{NO}_{\mathrm{x}}\right)$, Alcohol, Benzene, Smoke and carbon dioxide $\left(\mathrm{CO}_{2}\right)$. However, even though the MQ-135 is capable in detecting these various gases, it needs to be calibrated to accurately detect the presence of a certain type of gas. Different gas will have a different equation and conversion parameters in order to know the concentration of a particular gas in ppm (parts-per-million). However, in this study, the aim is not to accurately know the concentration of a particular gas, but to give warning if there is presence of any harmful gases. Therefore, the output value of the MQ-135 sensor is simply converted into a percentage conductivity, whereby the higher percentage of conductivity reflects a high harmful gas concentration in the surrounding.

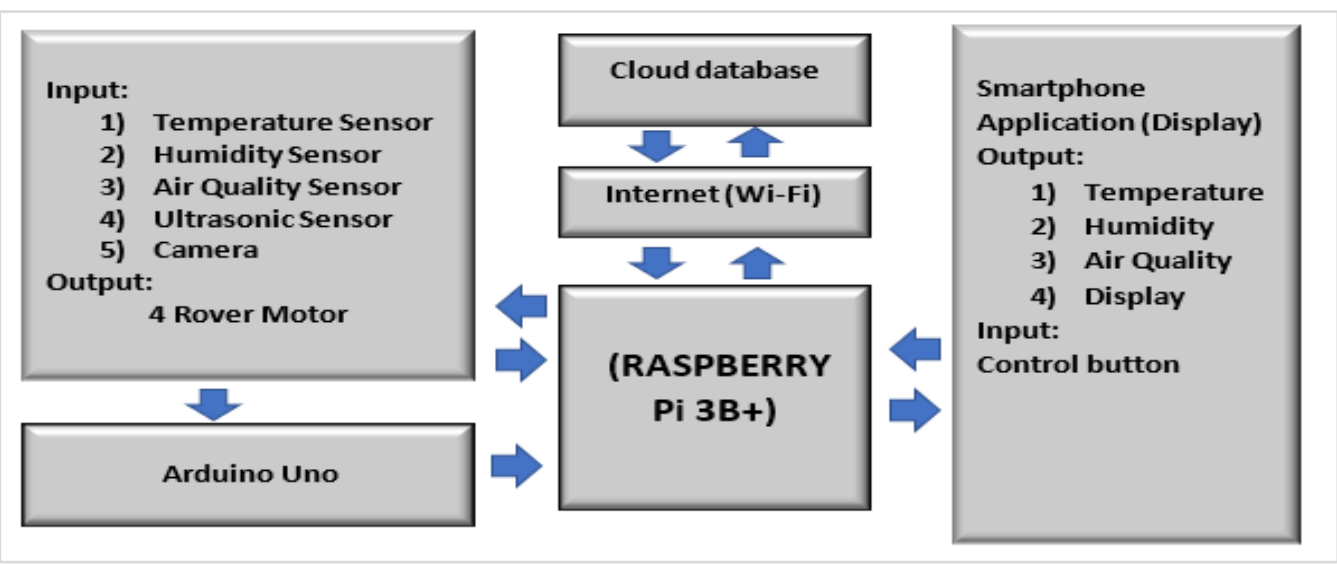

Figure 1 Block diagram of the overall system architecture of the IoT based environmental monitoring rover

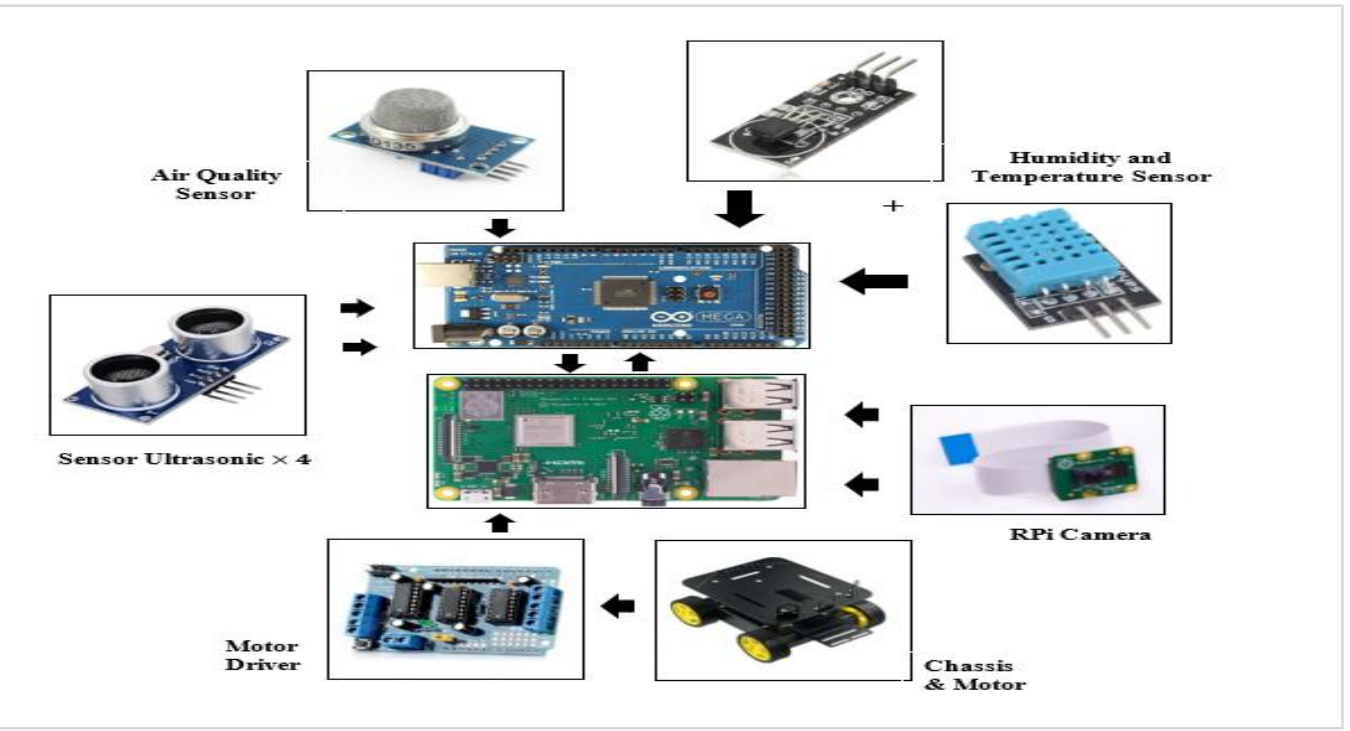

Figure 2 Hardware of the environmental monitoring rover 
Table 1 Cost of each component of the rover

\begin{tabular}{ll}
\hline Item & Cost (RM) \\
\hline Ultrasonic sensor & 3.30 \\
\hline MQ135 Sensor & 10 \\
\hline DHT-11 Sensor & 6.50 \\
\hline Arduino & 39 \\
\hline Car Chasis + Motor & 216.6 \\
\hline Raspberry Pi & 160 \\
\hline Pi Camera & 19.50 \\
\hline Total & $\mathbf{R M ~ 4 6 2 . 4}$ \\
\hline
\end{tabular}

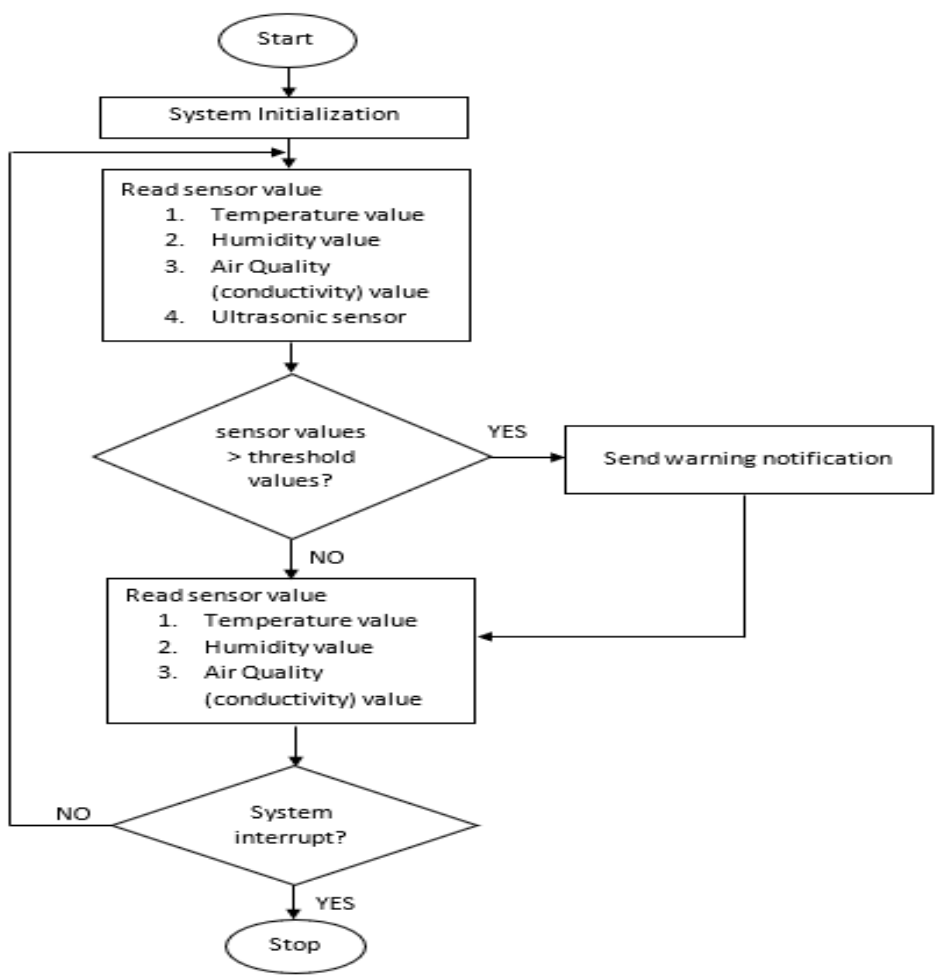

Figure 3 The flowchart of the rover system

\subsection{Software}

The main programming of this study comprises sending data from sensors to cloud databases, sending data from cloud to site and using PHP programming, and Java programming to develop mobile phone applications capable of retrieving data and displaying the data as well as being able to send data to database. Data from sensors are sent to cloud (MySQL) database and can be accessed at any time for further analysis. Android Studio was used to develop the smartphone apps. The reading of sensor and motor control algorithm within the Raspberry Pi is written with Python language. The overall flow of the system is as shown in Figure 3. The system will continuously read the sensor values until there is a 67 system interrupt, such as reset and/or power button has been pressed. Whenever there are values that exceeded any of the set threshold, notification will be sent to the user as a warning. The threshold set for temperature, humidity, air quality (conductivity) and distance from obstacles are, $40^{\circ}, 90 \%, 40 \%$ and $5 \mathrm{~cm}$ respectively.

The rover and smartphone app can only be used when both are connected under the same Wi-Fi. This is because the server and the database have been downloaded in the Raspberry-Pi used in the Rover development. Therefore, when the smartphone app is not connected to Raspberry-Pi / Rover, the database and server are inaccessible. Therefore, the 
smartphone app is unable to change the contents of the table in the database to control the rover nor retrieve data from the database to be displayed in the smartphone app, as well as access the display on the website that showcases the camera's visuals.

\section{Results and discussion}

Figure 4 illustrates the prototype of the proposed rover with (left) and without (right) the enclosed cover, and Figure 5 shows the 3D design of the
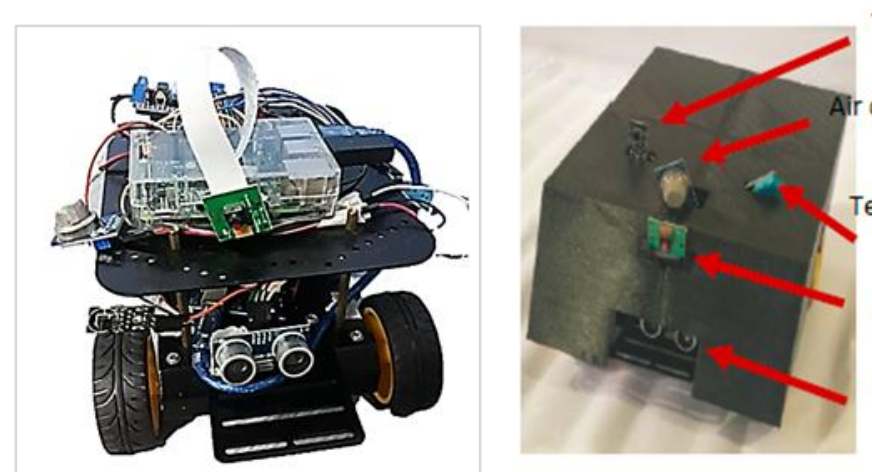

Figure 4 Prototype of the environmental monitoring rover
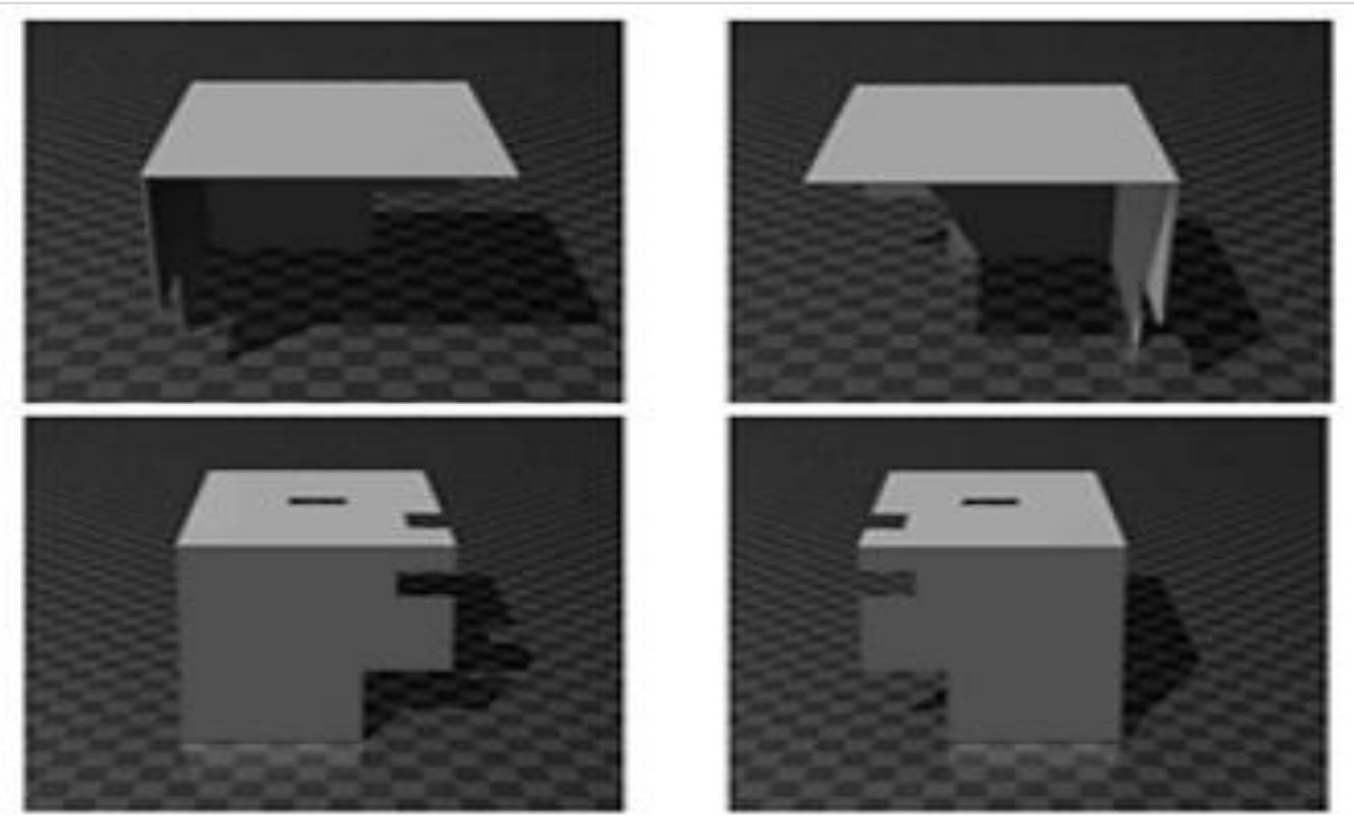

Figure 5 3D CAD drawing of the protection cover of the rover

\subsection{Air quality monitoring}

Experiment was performed using two different types of materials which are nail polisher and cigarette lighter. Nail polisher is an alcohol-based ingredient and cigarette lighter releases butane gas when is it light up. The rover was controlled to approach the gas sources (separately) at three different distances, 68

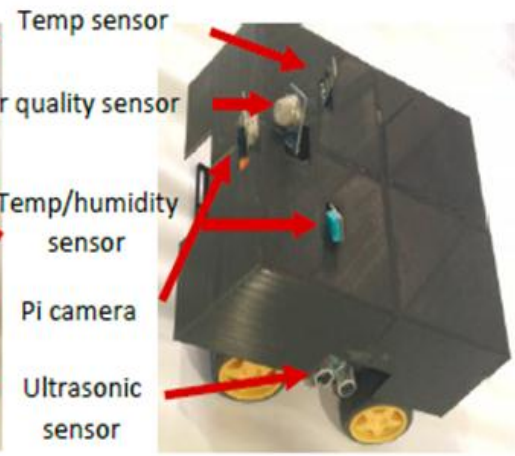

protection cover of the rover and to secure the camera position of the rover. The $3 \mathrm{D}$ design software used for the drawing is Blender. The chassis of this prototype was used to gather data and conduct the monitoring of several environmental surroundings.

All the sensors onboard were calibrated before the rover was assembled.

sensor

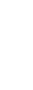


distance. The MQ-135 air quality sensor readings of the experiment conducted are as shown in Figure 6. The test was done in an enclosed room with the fan switched-off to obtain more accurate air quality readings. The graph shows that percentage of conductivity will increase when the distance of the gas source is closer.

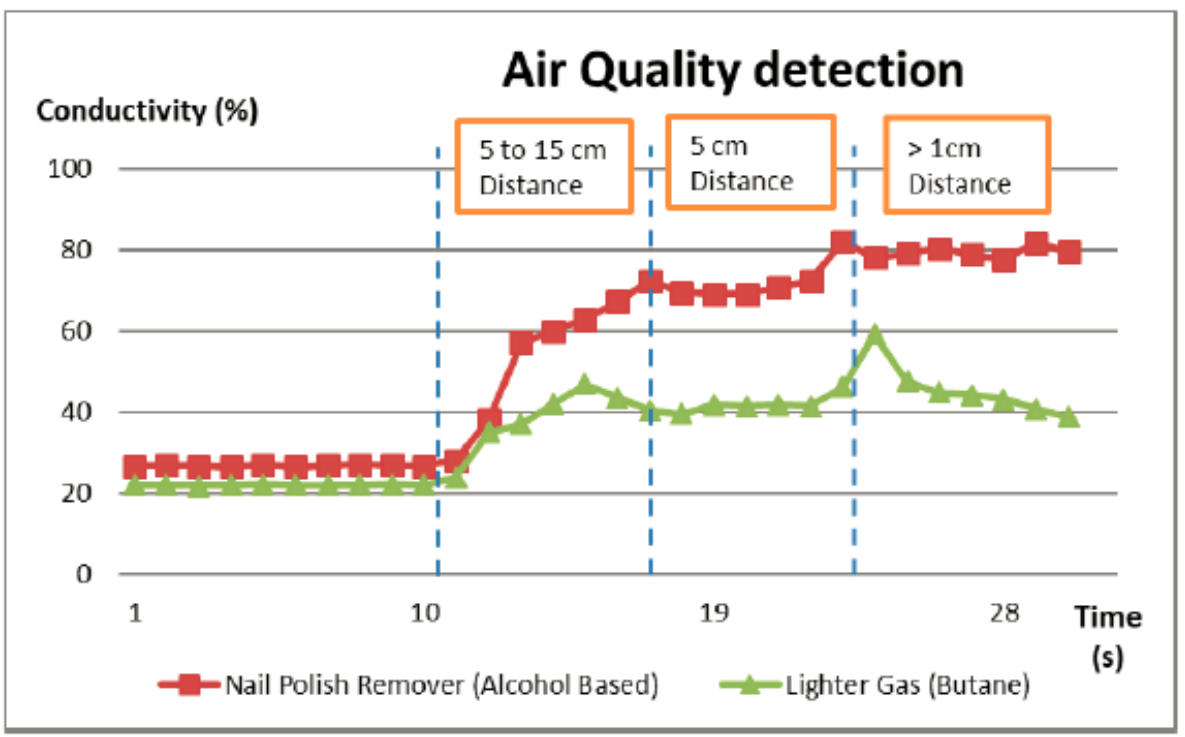

Figure 6 Air quality sensor reading from the MQ-135 sensor

\subsection{Temperature and humidity monitoring} Based on the datasheet, Humidity and Temperature Sensor DHT11 is able to detect relative humidity of $20-90 \%$ and temperature from $0-50{ }^{\circ} \mathrm{C}$. The rover was controlled to roam around the Faculty of Engineering and Built Environment (FKAB), UKM at $2.30 \mathrm{pm}$ to collect the humidity data of the faculty and the result is as shown in Figure 7.

DHT-11 also provide temperature value and the temperature value was acceptable as well. However, the DHT 11 has a limited temperature range. Because one of the focus of application of this rover is to monitor the area of radiation-risk facilities/surroundings, and there is a possibility for a high temperature fluctuation in the area, therefore, temperature range detected by the DHT-11 sensor is not suitable. Thus, the DS18B20 Temperature Sensor is also mounted on the rover, and has been used due to its temperature detection range between $-55^{\circ} \mathrm{C}$ to $135^{\circ} \mathrm{C}$.

\subsection{Mobile application}

The smart phone app used in this system has been developed using Android Studio. The mobile app interfaces are shown in Figure 8. The developed application comes with a button for steering the rover movement towards the FRONT, BACK, LEFT and RIGHT. It also comes with a 'connect' button for viewing from the video from the camera and displaying it on the interface. The pi camera managed to provide a satisfaction quality of resolution for the user to monitor the site. The rover can still be controlled if the camera is not displayed on the apps itself. Camera control button and display button are placed in the same interface as the rover movement buttons to help users to move the rover and monitor the surroundings to facilitate mobility, as well as for visual monitoring. Then the 'sensor data' button, once clicked, leads to the next interface, which displays the data from the sensors used, in a timely manner. Data from sensors can help in monitoring the environment parameters. 
Nurul Ayni Mat Pauzi et al.

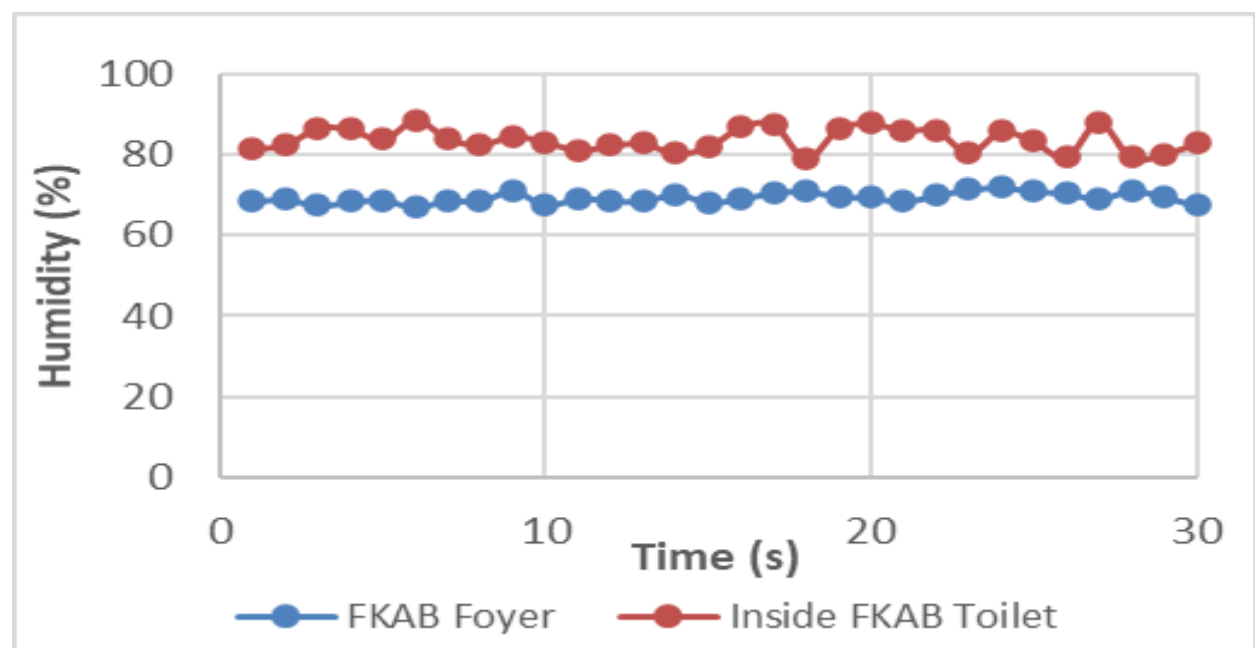

Figure 7 Humidity sensor reading from the DHT-11 sensor

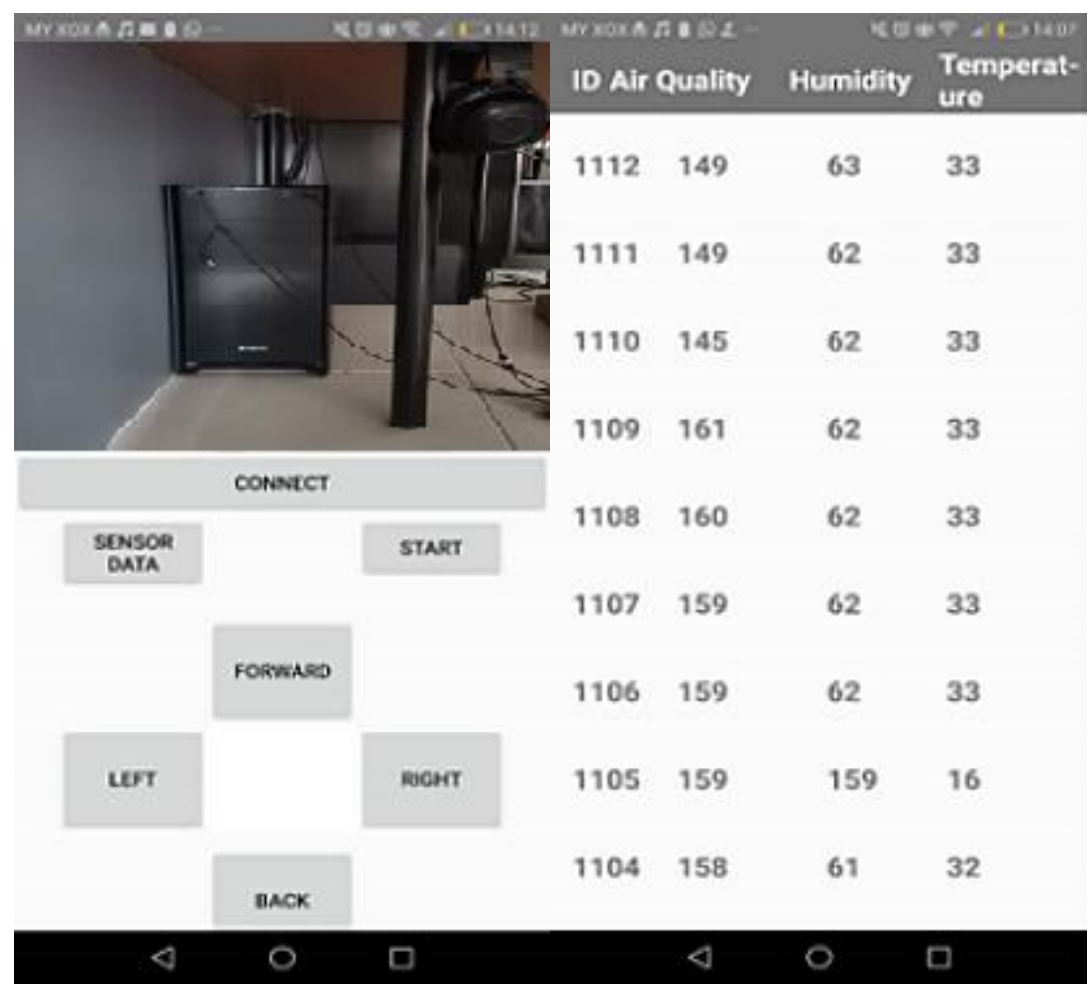

Figure 8 The developed mobile apps user interface

\section{Discussion}

Based on the sensor reading of air quality at the two tested location, the closer the rover is to the source of the pollution, the higher percentage of conductivity due to the high concentration of toxicity. The further away the rover form the source, the lower the conductivity value. The value from 10 to $15 \mathrm{~cm}$ reading is roughly the same because the alcohol and butane has dispersed to the air making the concentration lower. Further than $15 \mathrm{~cm}$, the sensor can still detect some percentage of pollution in the air, which was about $20 \%$ of the gas toxicity that was detected. This experiment shows the importance of having a mobile rover to carry out hazardous environment monitoring because if human were to carried out the task, the person needs to be very close to the source of the toxic gas to detect location of gas leakage or toxic waste. Humidity sensing feature of 
this rover contributed by the DHT-11 sensor, was satisfying as compared to the typical Malaysia humidity data provided by www.timeanddate.com, whereby the humidity should be between $65 \%$ to $85 \%$ depending on the location and time of day. In the case of the experiment, the humidity was $72 \%$ at an unenclosed spaced (faculty foyer) and at an average of $86 \%$ in the faculty's restroom. Temperature of the surrounding can also be monitored, and can be viewed via the mobile application developed specific for the rover.

\subsection{Limitation}

Major limitation to the current system is the battery life. The system is design to be light weight and have very minimal feature, but at the same time capable to perform basic monitoring, to avoid consuming too much power from the battery. At the moment, the system is utilizing $12 \mathrm{~V}$ AA battery abd only capable of 10 to 15 minutes monitoring runtime, at an average of $80 \%$ PWM. This means that the system, and monitoring distance very much dependent on the capabilities of the battery. The system also is lacking a sensor that is capable in detecting any form of ionizing radiation such as Geiger counter, an important feature if the rover is to be used to monitor hazardous environment.

\section{Conclusion}

The Environmental Monitoring Rover based on IoT technology has been successfully developed. The Rover is equipped with four types of sensors to detect temperature, humidity and air quality sensors parameters such as DS18B20 temperature sensors, DHT11 humidity sensors and MQ-135 air quality sensors as well as sensors to realize autonomous basics by detecting obstacles in front, rear, left and right of the rover, 4 HC-SR04 ultrasonic sensors. The sensors used have been tested and undergo calibration. All the monitoring sensors chosen performed well and suitable to be used for the application of interest. The sensors, cameras and motors are connected to the Raspberry $\mathrm{Pi}$, applying an IoT technology making monitoring the environmental condition of a confined and tight space simple and easy for area such as the ventilation duct and system, which are difficult to monitor.

For future improvement of this environmental monitoring rover, autonomous movement capabilities to the rover system will be added on top of the manual control. This will enable the system to become a partial unmanned system where monitoring can be performed without supervision. The rover function will be diversified and optimized by adding other sensors suitable for monitoring hazardous environment such as an Arduino-based Geiger counter which is capable in detection ionizing radiation.

\section{Acknowledgment}

The work described in this article was supported by the Young Researcher Grant (GGPM), of Universiti Kebangsaan Malaysia under grant no GGPM-2019-060.

\section{Conflicts of interest}

The authors have no conflicts of interest to declare.

\section{References}

[1] Panfil W, Przystałka P, Adamczyk M. Behavior-based control system of a mobile robot for the visual inspection of ventilation ducts. In recent advances in mechatronics 2007 (pp. 62-6). Springer, Berlin, Heidelberg.

[2] Nguyen L, Kodagoda S, Ranasinghe R, Dissanayake G. Mobile robotic sensors for environmental monitoring using gaussian Markov random field. Robotica.:1-23.

[3] Noda K, Aizawa H. Indoor environmental monitoring system using a robot vacuum cleaner. Sensors and Materials. 2020; 32(3):1133-40.

[4] Raj AH. Development of an intelligent sensor based inspection robot for closed environment. In Nirma university international conference on engineering 2015 (pp. 1-6). IEEE.

[5] Kurkin AA, Tyugin DY, Kuzin VD, Chernov AG, Makarov VS, Beresnev PO, et al. Autonomous mobile robotic system for environment monitoring in a coastal zone. Procedia Computer Science. 2017; 103:459-65.

[6] Yamazaki Y, Nomura K, Saito T, Tatsuno K, Sota K, Fuziwara Y, et al. Remote control console of mowing robot with image monitor. In international symposium on micro-nanomechatronics and human science 2017 (pp. 1-5). IEEE.

[7] Kafumbe S. iRovers: real-time unmanned four-wheel IoT vehicles for air and noise pollution monitoring. In advances in science and engineering technology international conferences 2018 (pp. 1-5). IEEE.

[8] Saat S, Kamil AR, Tumari MZ, Subki AS. Development of an autonomous robot for inspection system. In international colloquium on signal processing \& its applications 2018 (pp. 272-6). IEEE.

[9] Razak AA, Abdullah AH, Kamarudin K, Bakar MA, Salih JE, Ilias B, et al. Development of mobile robot in confined space application. In 13th international colloquium on signal processing \& its applications 2017 (pp. 190-5). IEEE.

[10] Salman H, Rahman MS, Tarek MA, Wang J. The design and implementation of GPS controlled environment monitoring robotic system based on IoT 
Nurul Ayni Mat Pauzi et al.

and ARM. In international conference on control and robotics engineering 2019 (pp. 93-8). IEEE.

[11] Singh P, Nigam P, Dewan P, Singh A. Design and implementation of a raspberry pi surveillance robot with pan tilt Raspbian camera. International Journal of Nanotechnology and Applications. 2017; 11(1):69-73.

[12] Wahed MA, Arshad MR. Wall-press type pipe inspection robot. In international conference on automatic control and intelligent systems 2017 (pp. 185-90). IEEE.

[13] Devi NS, Raju KS, Madhu A, Sekhar RR. Safety and security for school children's vehicles using GPS and IOT technology. International Journal of Advanced Trends in Computer Science and Engineering. 2018; 7(6):91-5.

[14] Sunitha M, Vinay PD, Lokesh VS, Kumar BD. IP based surveillance robot using IOT. In 2020 fourth international conference on I-SMAC (IoT in Social, Mobile, Analytics and Cloud) (I-SMAC) 2020 (pp. 337-42). IEEE.

[15] Rao SV, Devi MS, Kishore AR, Kumar P. Wireless sensor network based industrial automation using internet of things (IoT). International Journal of Advanced Trends in Computer Science and Engineering. 2018; 7(6):82-6.

[16] Das A, Sarma MP, Sarma KK, Mastorakis N. Design of an IoT based real time environment monitoring system using legacy sensors. In MATEC web of conferences 2018. EDP Sciences.

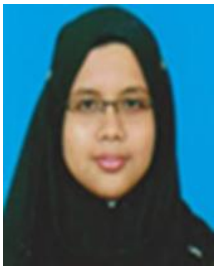

Nurul Ayni Mat Pauzi received his B. Eng from the Universiti Kebangsaan Malaysia in Electrical and Electronics Engineering in 2019. His research interest is in the area of System Engineering. She is currently a research assistant in UKM, Malaysia.

Email: ayni.pauzi@siswa.ukm.edu.my

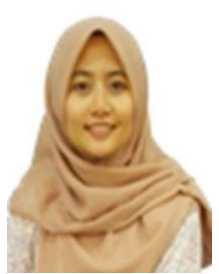

Seri Mastura Mustaza (Dr.) received her B.Eng. in Biomedical Engineering from the Vanderbilt University, TN, USA, and M.Sc. in Electrical Engineering (Mechatronics and Control) from Universiti Teknologi Malaysia, in 2006 and 2011, respectively. She received her $\mathrm{PhD}$ in Electronics Engineering from University of Surrey in 2018. Currently, she is a senior lecturer in UKM and current research interest is in the field of Control and Robotics Engineering, and Intelligent System.

Email: seri.mastura@ukm.edu.my

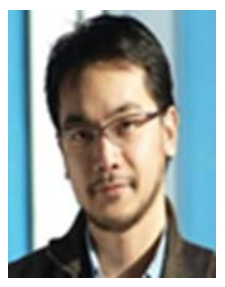

Iskandar Yahya (Dr.) received his M.Eng. in Electrical and Electronics, from University of Sheffield, UK. In 2013, he received his $\mathrm{PhD}$ from University of Surrey. He is currently a senior lecturer at the Faculty of Engineering and Built Envuronment, UKM. His current research interest is in the field of Semiconductor Technology and Devices, Nanoelectronics and Advanced Carbon-based Devices.

Email: iskandar.yahya@ukm.edu.my 